\title{
Configuration of a newly optimized multi-cyclones unit as a fine particulate emission separator in air pollution control
}

\author{
Norelyza Hussein ${ }^{a,}{ }^{*}$, Mohd Rashid Mohd Yusof ${ }^{b}$, Nur Hasyimah Hashim ${ }^{b}$, Eeydzah Aminudin ${ }^{a}$, \\ Che Hafizah Che Hassanc
}

a School of Civil Engineering, Faculty of Engineering, Universiti Teknologi Malaysia, 81310, Johor Bahru, Johor, Malaysia

b Air Resources Research Laboratory, Malaysia-Japan International Institute of Technology (MJIIT) 54100 UTM Kuala Lumpur, Malaysia

${ }^{c}$ Faculty of Chemical and Energy Engineering (FKT), Universiti Teknologi Malaysia, 81310, Johor Bahru, Johor, Malaysia

* Corresponding author: norelyza@utm.my

\section{Article history}

Received 12 January 2019

Revised 5 May 2019

Accepted10 October 2019

Published Online 2 February 2020

\begin{abstract}
Multi-cyclones separator, which consists of many miniature cyclones, works in the same principle as single cyclone in separation of particulate matter from flue gas. However, multi-cyclone is able to attain higher collection efficiency and concurrently avoid rapid increasing of pressure drop due to the usage of small diameter cyclone. The studies on multi-cyclones are very limited and lacking especially on its design configurations due to its confidentiality and commercial reason. Thus, a configuration of a newly optimized multi-cyclone unit named as MR-deDuster is discussed and assessed in this study. Six dimensions considered in the study include diameter of cyclone $(D)$, diameter of vortex finder $\left(D_{e}\right)$, length of cyclone body $\left(L_{b}\right)$, length of cyclone cone $\left(L_{c}\right)$, length of vortex finder $(S)$, and diameter of dust outlet $\left(D_{d}\right)$. The theoretical background of the unit was developed based on the modifications of established design equations available in literatures. The selection of the new dimension and the actual size of the unit were based on two main criteria (the performance of the unit based on its cut-diameter and the ratio of axial dimensions). The predicted cut-diameter and pressure drop of the selected dimension was $1.7 \mu \mathrm{m}$ and $86 \mathrm{~mm}$ of water, respectively. Meanwhile, the optimum axial ratios of the final design were $L_{b} / D=1.6, S / D=1$, and $L_{b}-S / D=0.7$, with respect to the diameter of the cyclone.
\end{abstract}

Keywords: Air pollution, multi-cyclones, multi-cyclones configurations, multi-cyclones design, particulate control, particulate emission

() 2020 Penerbit UTM Press. All rights reserved

\section{INTRODUCTION}

Cyclone is one of the oldest types of particulate control equipment and mostly used for removing industrial particulate from air or processed gas. It is one of the most widely used separators primarily because of its geometrical simplicity, inexpensive economic value, have no moving part, and ability to work in harsh operating condition [1-2]. Cyclones come in numerous variations of designs, sizes, and shape, from complex arrangements of multiple small cyclones (i.e. multi-cyclones) to single large units of various configuration. Cyclone can be highly efficient but usually are medium-low efficiency which not adequate to meet stringent air pollution regulations [3], but ideal as pre-cleaner for more expensive final control devices. In order to obtain higher collection efficiency, it is suggested to use small-diameter cyclone. However, the small cyclone has a higher pressure drop and impractical to treat high volumetric flow rate of flue gas [4-5]. To overcome the problem, the modifications of cyclone design was investigated to obtain ideal configurations which is high in collection efficiency with considerably low pressure drop.

Studies have been conducted to enhance the performance of a cyclone by evaluating the effects of configurations and operational parameters [6-7]. Most studies reported that the configurations of a cyclone significantly affect its performance [8-12]. Also, configurations of the cyclone affect the flow of the fluid within the cyclone which give significant effect to the centrifugal and drag force of particulate. The efficiency of a cyclone increases as the cyclone body diameter decreases $[10,11,13,14]$. However, the pressure drop of a cyclone increases significantly if the diameter of a cyclone is too small. Studies have also shown the axial dimensions such as cyclone cylinder length, cone length, and vortex finder length affect the performance of a cyclone, whereas the collection efficiency appears to decrease to a certain extent if the cyclone length increases excessively $[8,12,13]$. In addition, studies reported by Elsayed and Lacor [15] and Hsu et al. [8] found that decreasing the cyclone entry area results in a smaller cut diameter which consequently increases the performance of the cyclone.

In this study, cyclone configurations such as cyclone body diameter $(D)$, vortex finder diameter $\left(D_{e}\right)$, cone tip diameter $\left(D_{d}\right)$, cyclone cylinder length $\left(L_{b}\right)$, cone length $\left(L_{c}\right)$, and vortex finder length $(S)$ parameters (as shown in Fig. 1) were considered in proposing a new multi-cyclone unit for fine particulate emission control named as MRdeDuster. The proposed unit was configured by theoretically optimizing these six parameters of a single cyclone initially. Then, these cyclones were arranged in parallel as a multi-cyclone unit characterized with high collection efficiency and low pressure drop. 


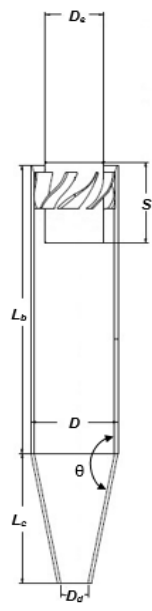

Fig. 1 Configurations of cyclone with axial entry.

\section{METHODOLOGY}

Initially, the design of MR-deDuster was determined by identifying the dimensions of a single prototype unit. The actual size of single prototype unit that adopted the optimum dimensions was later selected. The development of a single prototype unit is based on cyclone dimensions introduced by Lapple [9], Stairmand [16], and Swift [17] Six new set dimensions were generated using mathematical modification of these established dimensions. The optimum dimension was selected among these six new dimensions.

In the selection of optimum dimension, the diameter of cyclone was fixed and five other parameters (diameter of vortex finder, length of cyclone body, length of cyclone cone, length of vortex finder, and diameter of dust outlet) were classified as dependent variables. The trial and error approach which considered cut diameter, collection efficiency and pressure drop, as well as optimum axial dimensions were used to obtain the optimum dimensions.

In this study, modified cut diameter $\left(d_{p c}\right)$ equation based on Lapple [9] approach that re-arranged by Norelyza and Rashid [18] as well as original Lapple's fractional and overall collection efficiency were used to predict the collection efficiency of the six new set dimensions. Meanwhile, the modified Sherperd and Lapple [19] approach was used to calculate the pressure drop. The calculation was based on common cyclone inlet velocity which is $15 \mathrm{~m} / \mathrm{s}$ [4]. Meanwhile, ambient temperature was used as temperature reference and actual particulate size distribution of palm oil mill fly ash (POFA) was used as particulate size distribution reference. The diameter of cyclone body used for the calculation was fixed at $150 \mathrm{~mm}$ based on the smallest diameter used for multi-cyclone tube that was reported by previous literature [4].

$$
d_{p c}=\left[\frac{9 \mu\left(D-D_{e}\right)}{4 \pi N_{e} v_{i}\left(\rho_{p}-\rho_{g}\right)}\right]^{1 / 2}
$$

where $\mu$ is the gas viscosity, $\mathrm{N}_{\mathrm{e}}$ is the number of effective turns, $v_{i}$ is the gas inlet velocity, $\rho_{\mathrm{g}}$ is the density gas, and $\rho_{\mathrm{p}}$ is the density of particulate.

$$
\begin{aligned}
& \eta_{j}=\frac{1}{1+\left(d_{p c} / d_{p j}\right)^{2}} \\
& \eta_{o}=\Sigma \eta_{j} m_{j}
\end{aligned}
$$

where $\eta_{j}$ is collection efficiency for the $j$ th particulate size range, $d_{p c}$ is the cut diameter, $d_{p j}$ is the characteristic diameter of $j$ th particulate size range, $\eta_{o}$ is the overall collection efficiency, and $m_{j}$ is the mass fraction of particulate size range.

$$
\begin{gathered}
H_{v}=\frac{K \pi\left(D^{2}-D_{e}^{2}\right)}{4 D_{e}^{2}} \\
\Delta P=\frac{1}{2} \rho_{g} v_{i}^{2} H_{v}
\end{gathered}
$$

where $H_{v}$ is pressure drop, expressed in number of inlet velocity head, $\Delta P$ is pressure drop, $\rho_{g}$ is the gas density, $v_{i}$ is the gas inlet velocity, and $K$ is a constant that depends on cyclone configuration and operating condition. $K$ value is set equal to 16 based on Licht [10].

The axial dimensions range introduced by Zhu and Lee [12] as shown in Table 1 was referred to determine the optimum axial dimensions of new configurations.

Table 1 The optimum range of axial dimensions [12].

\begin{tabular}{cc}
\hline Dimensions & Optimum Range \\
\hline$L_{b} / D$ & $1.0-2.0$ \\
$S / D$ & $1.0-1.5$ \\
$L_{b}-S / D$ & $0.5-1.0$ \\
\hline
\end{tabular}

The determination of actual size of MR-deDuster unit adopted the selected dimensions among six new set dimensions. Further, four different diameters of cyclone body $(85 \mathrm{~mm}, 105 \mathrm{~mm}, 123 \mathrm{~mm}$, and $139 \mathrm{~mm}$ ) were tested in selection the optimum size of the unit. The selection of MR-deDuster size was strongly dependent on the selection of cyclone diameter while the other dimensions changed accordingly to the changes of the cyclone diameter. The trial and error method using cut diameter, fractional efficiency, and pressure drop as used in the selection of new dimensions were also used in size selection of MRdeDuster. In the size selection of MR-deDuster, the requirement of cut diameter is equal or lower than $2.5 \mu \mathrm{m}$ and the fractional efficiency are higher than $90 \%$ for $5 \mu \mathrm{m}$ and smaller particulates. The purpose of small cut diameter and higher fractional efficiency requirements is to fulfil the purpose of MR-deDuster design which is to control the emission of the fine particulates. The axial dimensions introduce by Zhu and Lee [12] as shown in Table 1 was also used in determining the diameter of MR-deDuster.

\section{RESULTS AND DISCUSSION}

\section{Dimensions of a single unit cyclone}

The development of the MR-deDuster is primary based on the dimensions of cyclone with tangential entry introduced by Lapple [9], Stairmand [16], and Swift [17]. However, the six set of established dimensions introduced by these authors were modified mathematically

\begin{tabular}{|c|c|c|c|c|c|c|}
\hline Dimension & 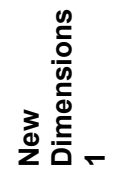 & 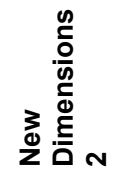 & 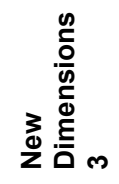 & 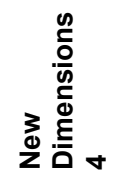 & 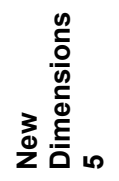 & 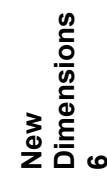 \\
\hline $\begin{array}{l}\text { Diameter } \\
\text { of Vortex } \\
\text { Finder, } D_{e}\end{array}$ & $0.5 D$ & $0.75 D$ & $0.4 D$ & $0.5 D$ & $0.75 D$ & $0.5 D$ \\
\hline $\begin{array}{l}\text { Inlet Area, } \\
A\end{array}$ & $\frac{\pi\left(D^{2}-D_{e}^{2}\right)}{4}$ & $\frac{\pi\left(D^{2}-D_{e}^{2}\right)}{4}$ & $\frac{\pi\left(D^{2}-D_{e}^{2}\right)}{4}$ & $\frac{\pi\left(D^{2}-D_{e}^{2}\right)}{4}$ & $\frac{\pi\left(D^{2}-D_{e}^{2}\right)}{4}$ & $\frac{\pi\left(D^{2}-D_{e}^{2}\right.}{4}$ \\
\hline $\begin{array}{l}\text { Length of } \\
\text { Body, } L_{b}\end{array}$ & $\frac{13.62 A}{D}$ & $\frac{7.27 A}{D}$ & $\frac{12.58 A}{D}$ & $\frac{12.73 A}{D}$ & $\frac{9 A}{D}$ & $\frac{13.33 A}{D}$ \\
\hline $\begin{array}{l}\text { Length of } \\
\text { Cone, } L_{c}\end{array}$ & $1.67 L_{b}$ & $1.67 L_{b}$ & $1.79 L_{b}$ & $1.14 L_{b}$ & $1.18 L_{b}$ & $L_{b}$ \\
\hline $\begin{array}{l}\text { Length of } \\
\text { Vortex } \\
\text { Finder, } S\end{array}$ & $\frac{L_{b}}{3}$ & $\frac{L_{b}}{1.71}$ & $\frac{L_{b}}{2.8}$ & $\frac{L_{b}}{2.92}$ & $\frac{L_{b}}{2}$ & $\frac{L_{b}}{3.2}$ \\
\hline $\begin{array}{l}\text { Diameter } \\
\text { of Dust } \\
\text { Outlet, } D_{d}\end{array}$ & $0.375 D$ & $0.375 D$ & $0.4 D$ & $0.4 D$ & $0.4 D$ & $0.25 D$ \\
\hline
\end{tabular}
to generate six new set dimensions of cyclone with axial entry. These new dimensions were considered for a single unit of MR-deDuster as listed in Table 2.

Table 2 New possible dimensions ratios of the MR-deDuster. 
Table 2 also shows that all dimensions are strongly dependent on the cyclone diameter $(D)$ which changes accordingly to the diameter of the cyclone. The diameter of vortex finder $\left(D_{e}\right)$ also plays an important role in the geometrical dimensions since the diameter of cyclone body and vortex finder are affecting the inlet area $(A)$ of the unit. Thus, the inlet area of new dimensions has a significant effect on the axial dimensions such as cyclone body length $\left(L_{b}\right)$, cone length $\left(L_{c}\right)$, and vortex finder length $(S)$.

However, final selection of the dimension of unit was mainly based on its performance and the ratio range of the axial dimensions. The former was characterized by the cut-diameter, fractional collection efficiency, and pressure drop. Meanwhile, the latter was on the ratio of cyclone body length to cyclone diameter $\left(L_{b} / D\right)$, vortex finder length to cyclone diameter $(S / D)$, and the ratio of $L_{b}-S$ to cyclone diameter ( $L_{b^{-}}$ $S / D)$.

Fig. 2 (a), (b), and (c) present the performance of six new dimensions which were considered as the dimensions to be adopted for the MR-deDuster. The requirement of cut-diameter for the selection is $2.5 \mu \mathrm{m}$ concurring with the main purpose of developing MR-deDuster as the fine particulates emission control cyclone. Thus, the dimensions that present lower cut-diameter less than $2.5 \mu \mathrm{m}$ were considered to be used as configuration of MR-deDuster.

As depicts in Fig. 2 (a), only Dimension 2 and 5 having a small cutdiameter of $2.5 \mu \mathrm{m}$ compared to the others dimensions. This is due to the bigger size of vortex finder which results in smaller entry area and smaller cross sectional of both dimensions compared to others. Hsu et al. [8] and Elsayed and Lacor [15] reported that the decreasing of cyclone entry area results in a smaller cut-diameter which consequently increases the collection efficiency. The small cross sectional and smaller entry area will lead to higher flow velocity while the volume of volumetric air flow remains the same [8]. A smaller entry will result in higher tangential velocity and greater centrifugal force acting on particulates in the gas stream $[8,10,11,12,13,14,20]$. Particulates collide with the cyclone wall more often that result in increasing of particulate collection efficiency. In addition, a smaller cross sectional area of cyclone entry helps in reducing the radial distance travelled by a particulate and hence, increase the collection efficiency of the unit even for a fine size particulate.

As reported by Cooper and Alley [4], the multi-cyclone usually able to collect particulate of $10 \mu \mathrm{m}$ in size or PM10 at $90 \%$ collection efficiency. Thus, the same requirement was considered in the selection of dimension of the MR-deDuster. Again, Fig. 2 (b) similarly shows that Dimension 2 and 5 were the only dimensions able to collect higher than $90 \%$ efficiency of particulate equal or less than $10 \mu \mathrm{m}$ in size. These findings concurred that a smaller cut diameter found in both dimensions 2 and 5 resulted in a higher collection efficiency for fine particulate size fraction.

Fig. 2 (c) depicts the pressure drop of all new set cyclone dimensions considered in the study which showed that both Dimension 2 and 5 predicted a lower pressure drop which less than $250 \mathrm{~mm}(10$ inches) compared to the others. A typical pressure drop of a cyclone varies from $50 \mathrm{~mm}$ to $250 \mathrm{~mm}$ of water [10-11]. Meanwhile, for multicyclones unit, the pressure drop is between $50 \mathrm{~mm}$ ( 2 inches) and 150 $\mathrm{mm}$ (6 inches) of water [11]. Fig. 2 (c) also shows that both Dimensions 2 and 5 are able to meet the requirement on the pressure drop. This is due to small ratio of area of inlet entry to area of outlet duct (vortex finder) of both dimensions which is an important parameter in determining the frictional losses or kinetic energy losses that a dominant source of pressure drop in cyclone $[4,10,21]$. In addition, both dimensions also have bigger vortex diameter dimensions compared to others which lead to small pressure drop. Karagoz et al. [7] and Xiang and Lee [22] reported that the pressure drop of cyclone decreases with the increasing of vortex finder diameter.

Studies have shown that varying the length of cyclone does not significantly affect the cut-diameter of the cyclone [20,23]. However, this finding was disagreed by other studies $[7,8,12,24]$. Hsu et al. [8] as well as Zhu and Lee [12] found that the cut-diameter decreases as cyclone length increases, and results in increasing of particulate collection efficiency. However, as the length increases further, the collection efficiency is decreases indicating the existence of optimum cyclone length.

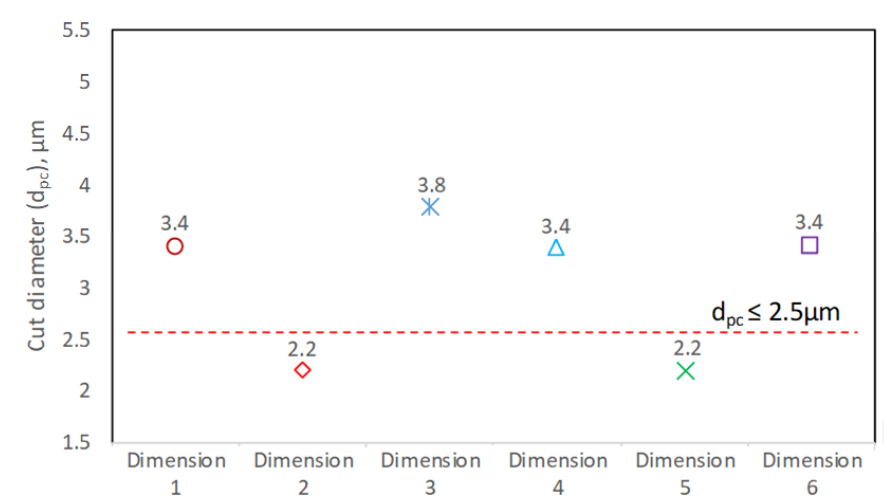

(a)

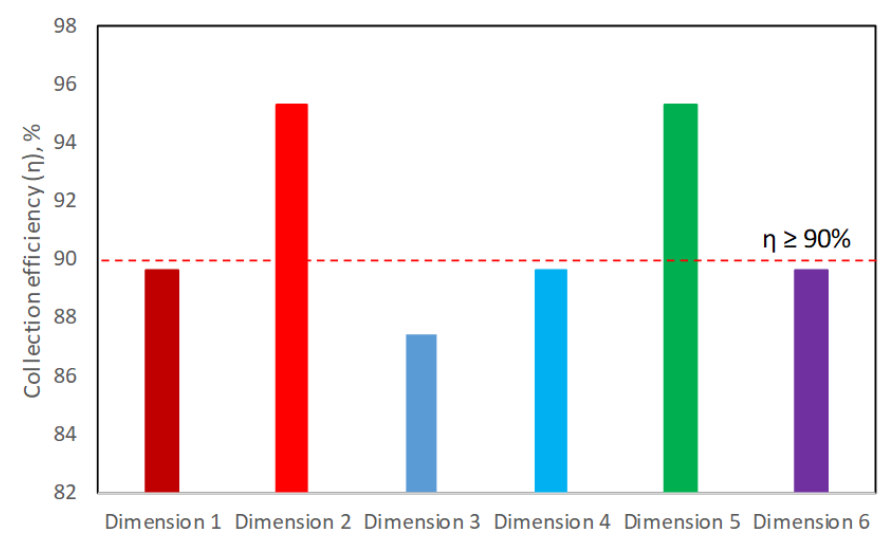

(b)

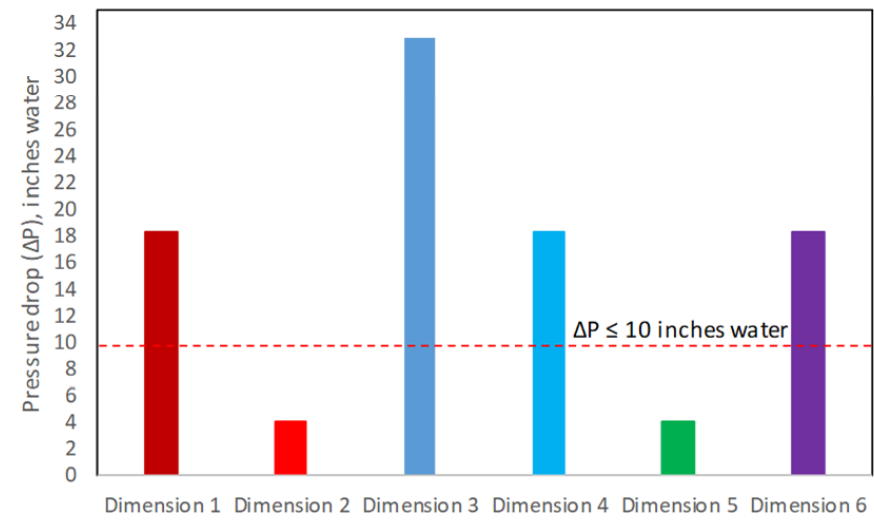

(c)

Fig. 2 The performance criteria of six new dimensions (a) cut diameter (b) fractional collection efficiency of PM10 (c) pressure drop.

The effect of cyclone length on the performance of cyclone are closely related to the natural vortex length $[8,12,24]$. Natural vortex length is referred to the depth where the gas vortex stream reverse from downward to upward direction [8]. There are several studies that have defined the formula of natural vortex length. Though, according to experimental study conducted by Zhu and Lee [12], the natural vortex length formula by Alexander [6] is more reasonable than other approaches. Zhu and Lee [12] claimed that if the cyclone length is shorter than Alexander's natural vortex length, the cyclone will not perform at its full separating potential, as the gas is forced into the inner vortex before it would have entered naturally. Meanwhile, if the cyclone length is longer than the natural vortex length, the gas stream will not reach the bottom of the cyclone and will create unutilized space. However, unutilized space served as a chamber to release gas pressure which aids in reduction of pressure drop in cyclone $[8,12]$. Thus, certain range of axial dimensions are needed in designing the cyclone in order to obtain optimum performance of the unit.

Figs. 3 (a), (b), and (c) illustrate the axial dimensions ratio of six new dimensions based on the study conducted by Zhu and Lee [12] by varying the axial dimensions to evaluate the particulate collection 
efficiency and pressure drop of small size diameter cyclone. The trend of the experimental results obtained in this study is consistent with other studies for optimized axial dimensions $[7,8,24,25]$.

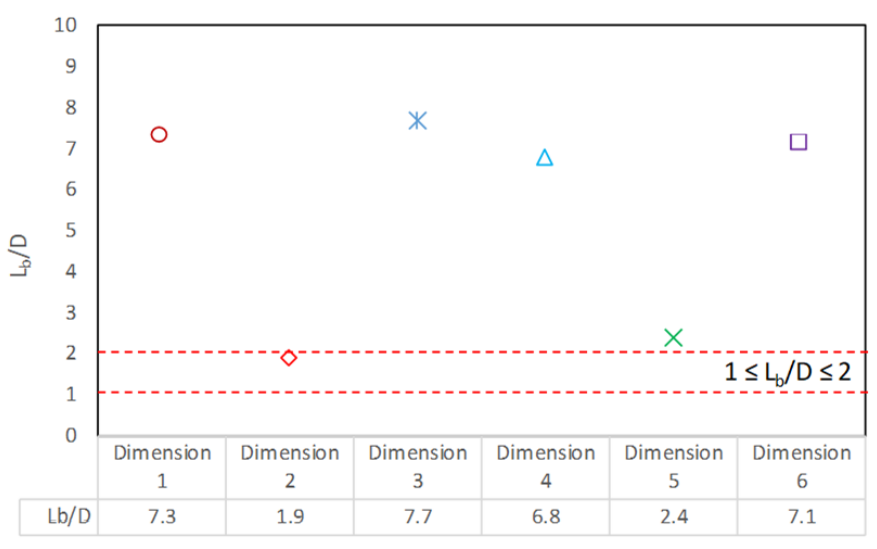

(a)

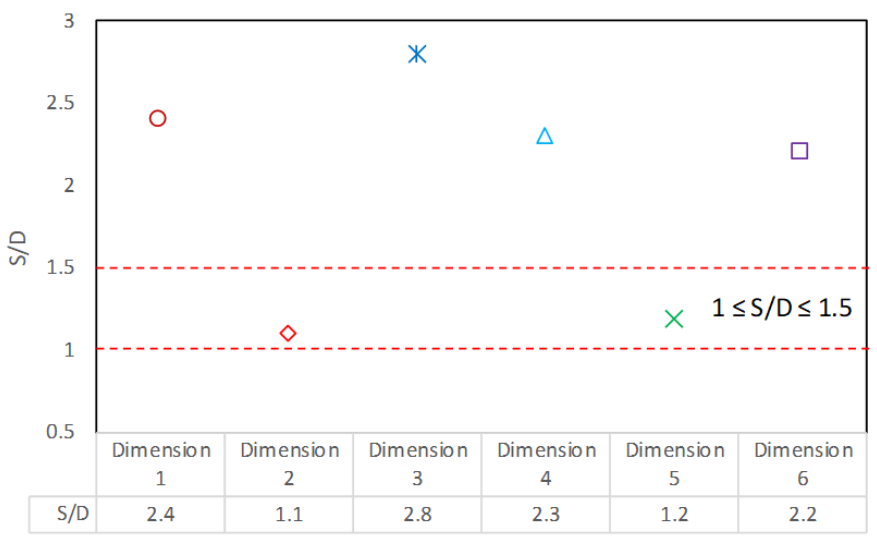

(b)

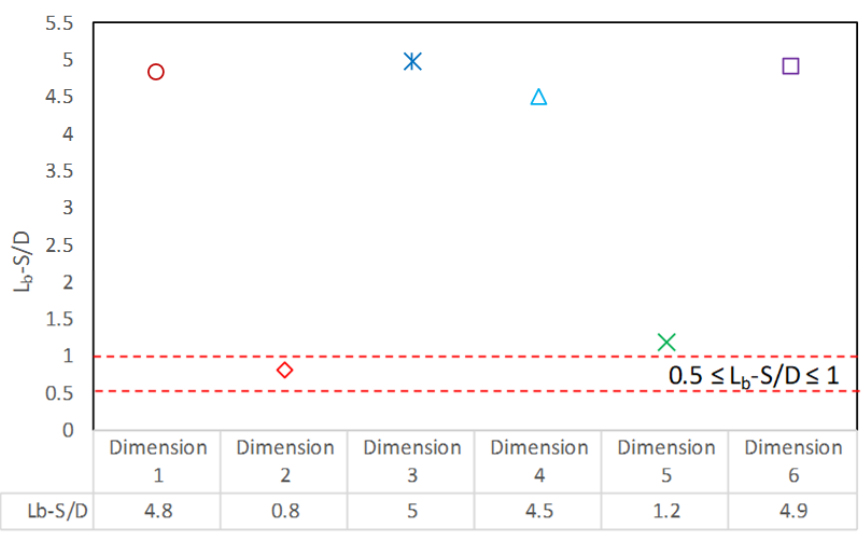

(c)

Fig. 3 The axial dimensions ratio of six new dimensions (a) $L b / D$ (b) $S / D$ (c) Lb-S/D.

Fig. 3 (a) presents the ratio of cyclone body length to cyclone diameter $(L b / D)$ of the six new dimensions. According to Zhu and Lee [12], the smallest cut diameter with considerable pressure drop was obtained when $L_{b} / D$ was between 1 and 2 . The authors reported that the pressure drop of the cyclone decreases substantially as the cyclone body is lengthened but the excessive length of cyclone body will result in lower collection efficiency. As shown in Fig. 3(a), only Dimension 2 has the value of $L_{b} / D$ which is within the range, while other dimensions have $L_{b} / D$ value more than 2 . This is because Dimension 2 has the smallest cyclone body length dimension $\left(L_{b}=7.27 \mathrm{~A} / D\right)$ compared to others (see Table 2). The other dimensions have exceeded the optimum cyclone body length requirement. Cyclone that is designed with shorter cyclone body length and longer cone body length will have better particulate collection efficiency $[8,12]$. This is due to more vortex migrate down to the cone section where the vortex can be accelerated due to decreased cross section area [12]. A small cross-sectional area will raise the tangential velocity and the centrifugal force of the particulate which eventually increases the collection efficiency. In addition, a decrease of collection efficiency after a certain height of cyclone body is due to fluid dynamics where the larger height result in lower energy swirl than the shorter height [8].

Fig. 3 (b) depicts the vortex finder length to cyclone diameter $(S / D)$ of the six new dimensions. The optimum range of $S / D$ is between 1 to 1.5 value and minimum cut diameter is obtained when the $S / D$ equal to $1[12,14]$. The figure shows that the $S / D$ value of Dimension 2 and Dimension 5 are within the range. However, the $S / D$ value of Dimension 2 is the nearest to 1 . The cut diameter is decreased and the particulate collection efficiency is increased when the vortex finder length is increased $[8,12,14,26]$. However, the particulate collection efficiency is decreased as the vortex finder length increases further. This is due to gas stream becomes overly pressed to the bottom part of the cyclone and decreasing the natural vortex length which results in increasing of cut diameter and increasing of collection efficiency [8]. The pressure drop of the cyclone also increase as the vortex finder length becomes longer $[8,12,14,26]$. This phenomenon is explained by higher kinetic energy losses between gas flow and vortex finder wall [14].

Fig. 3 (c) presents the ratio of $L_{b}-S$ to cyclone diameter $\left(L_{b}-S / D\right)$ of the six new dimensions. Zhu and Lee [12] stated that the axial dimensional value of $L_{b}-S$ is the most important axial dimension parameter determining the performance of a cyclone. This is because the relative small value of $L_{b}-S$ will cause most vortex to be present at the cone part of the cyclone where the most significant amount of deposition will take place [12,27]. The cone section also has smaller cross section area than the cylinder part which eventually will increase the flow velocity. The increasing of flow velocity will increase the centrifugal force and finally increase the particulate collection efficiency. However, the excessive cone length $\left(L_{c}\right)$ will reduce the collection efficiency of a cyclone due to increasing of cross section area $[8,12]$. The increasing of cross section area is due to larger $\theta$ (as shown in Fig. 1) which caused less gas streamline to reach the cone part and contribute to unutilized space. The minimal cut size is obtained when the $L b-S / D$ value is around 0.5 to 1.0 [12]. Fig. 3 (c) depicts that only $L_{b}-S / D$ value of Dimension 2 is between the range while the other dimensions exceed the value of 1.0 .

The Figs. 2 and 3 show that only Dimension 2 is able to meet both main requirements of the unit performance and the ratio range of axial dimensions. Therefore, it is concluded that the Dimension 2 is the most suitable dimension to configure the MR-deDuster.

\section{Dimension of MR-deDuster cyclone}

Four sizes of cyclone diameters were considered (i.e 85, 105, 123, and $139 \mathrm{~mm}$ ) before the finalizing the dimension of the MR-deDuster. The two main requirements are performance of the unit and the ratio range of axial dimensions that used in the selection of dimensions also used in selection of the actual size of MR-deDuster unit cyclone.

Figs. 4 (a), (b), and (c) present the performance of MR-deDuster with various cyclone diameters which showed that all cyclone diameters are able to meet the cut diameter criteria needed which are below and equal to $2.5 \mu \mathrm{m}$ (Fig. 4 (a)). However, only cyclones with diameters of 85 and $105 \mathrm{~mm}$ are able to attain $90 \%$ collection efficiency (Fig. 6(b)). The selection of PM5 fractional collection efficiency as a baseline in determine the cyclone diameter is based on the literature that reported the multi-cyclones is able to collect more than $90 \%$ for particulate of 5 to $10 \mu \mathrm{m}$ in size [4]. As for the pressure drop requirement, all cyclone diameters considered have pressure drop lower than $25 \mathrm{~cm}$ (10 inches) of water (Fig. 4 (c)). This is due to small ratio of area of inlet entry to area of outlet duct (vortex finder) of all diameters tested. 


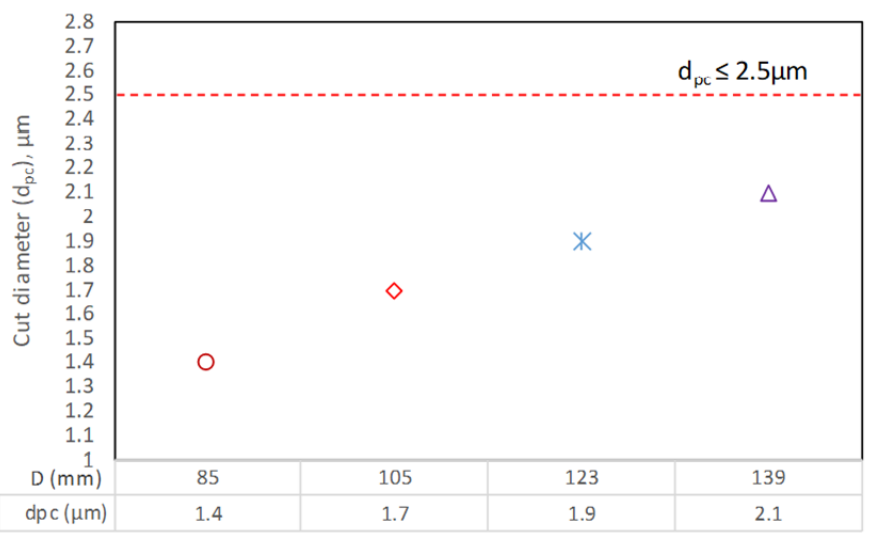

(a)

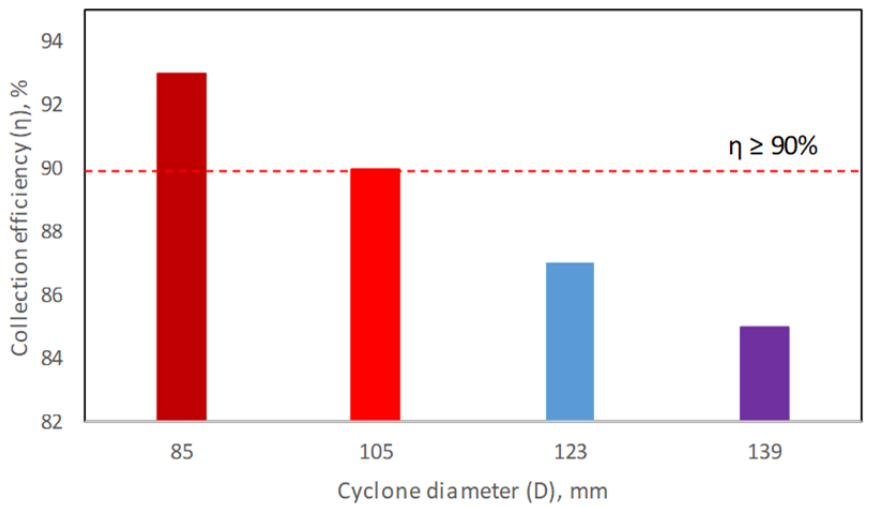

(b)

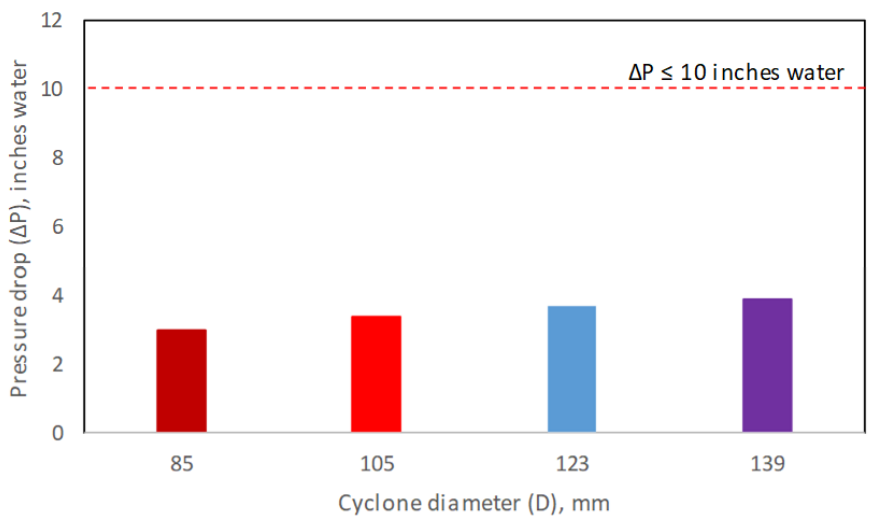

(c)

Fig. 4 The performance of MR-deDuster for different sizes of cyclone diameter (a) cut diameter (b) fractional collection efficiency of PM5 (c) pressure drop.

Figs. 4 (a) and (b) also illustrate relationship of cut diameter and collection efficiency on the different cyclone diameter. Fig. 4 (a) shows that the cut diameter is decreased with decreasing of cyclone diameter. Meanwhile, the PM5 fractional collection efficiency is increased with the decreasing of cyclone diameter. The finding is supported by the fractional collection efficiency of different cyclone diameter as illustrate in Fig. 5. In addition, Fig. 6 presents the relationship between cyclone diameter on overall collection efficiency and cut diameter. The figure portrays that the smaller cut diameter is obtained using the smaller cyclone diameter which finally will increase the collection efficiency of a cyclone.

The increasing of cyclone performance for smaller cyclone diameter is due to decreasing cross sectional area of the cyclone. The smaller cross-sectional area will result in higher tangential velocity and greater centrifugal force acting on particulates in the gas stream [1314]. The higher centrifugal forces on the particulates will result in more collision of particulates with the cyclone wall which lead to higher particulates collection [8]. Thus, particulate collection efficiency is increased even for finer particulate.

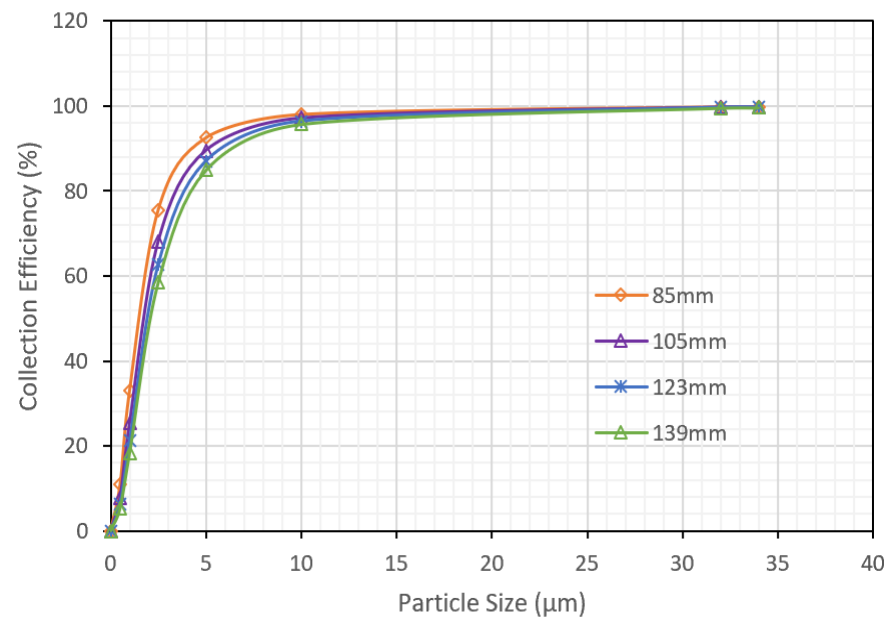

Fig. 5 Fractional collection efficiency of MR-deDuster for different cyclone diameters.

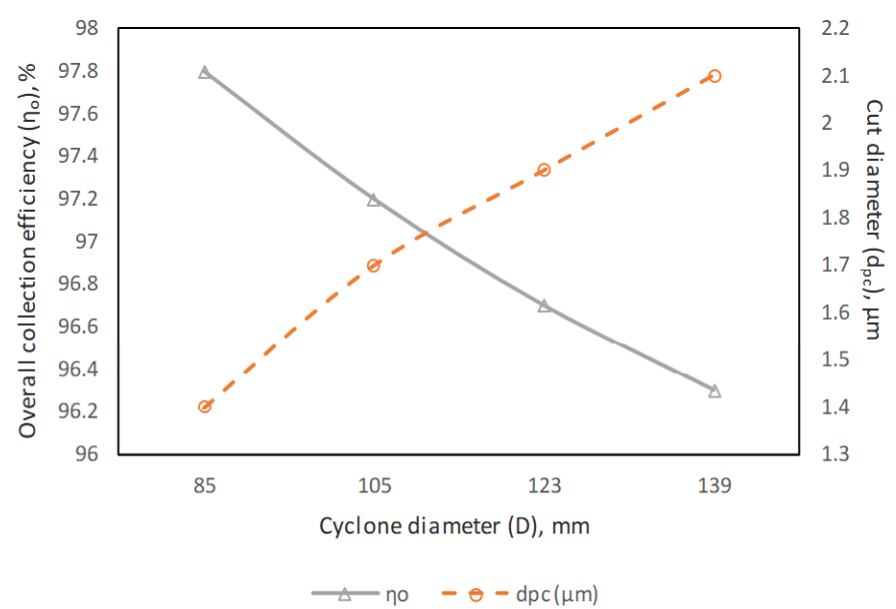

Fig. 6 Relationship between cyclone diameter on overall collection efficiency and cut diameter.

Figs. 7 (a), (b), and (c) present the axial dimensions ratio of different cyclone diameter sizes which show that cyclone diameter of 105,123 , and $139 \mathrm{~mm}$ are able to meet all the requirement of axial dimensions ratio. Meanwhile, cyclone with diameter of $85 \mathrm{~mm}$ only able to meet the range of $L_{b} / D$ and $L_{b}-S / D$. However, the cyclone configuration using cyclone diameter of $105 \mathrm{~mm}$ is the only configuration that is able to meet all of the requirements needed in size selection of MR-deDuster unit cyclone. Thus, the diameter and proto-type of MR-deDuster was finally fixed at $105 \mathrm{~mm}$ with its other configurations following on Dimension 2.

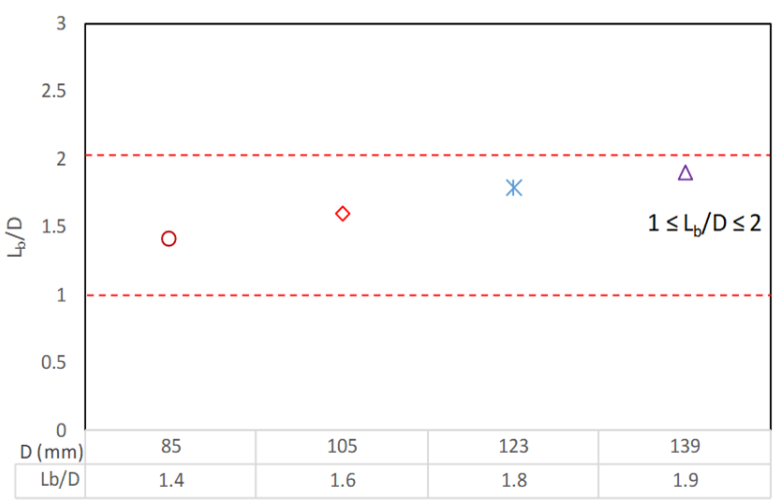

(a) 


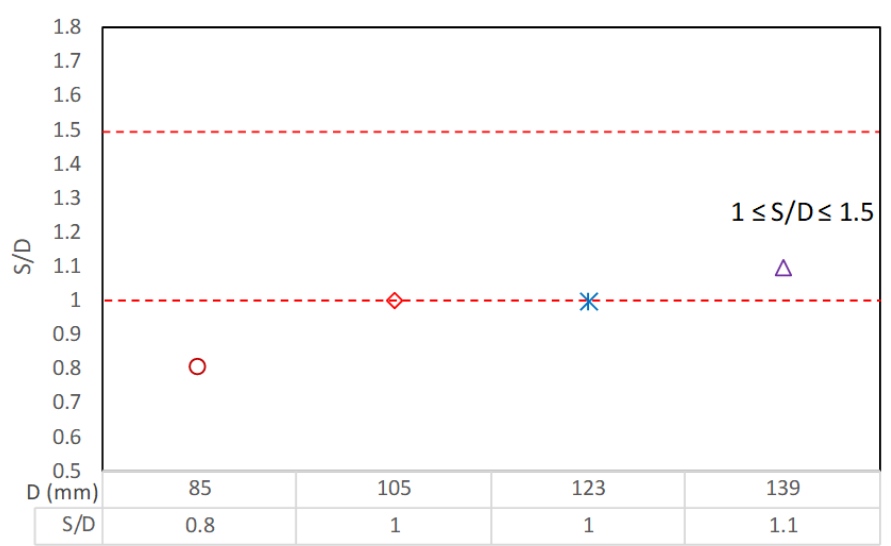

(b)

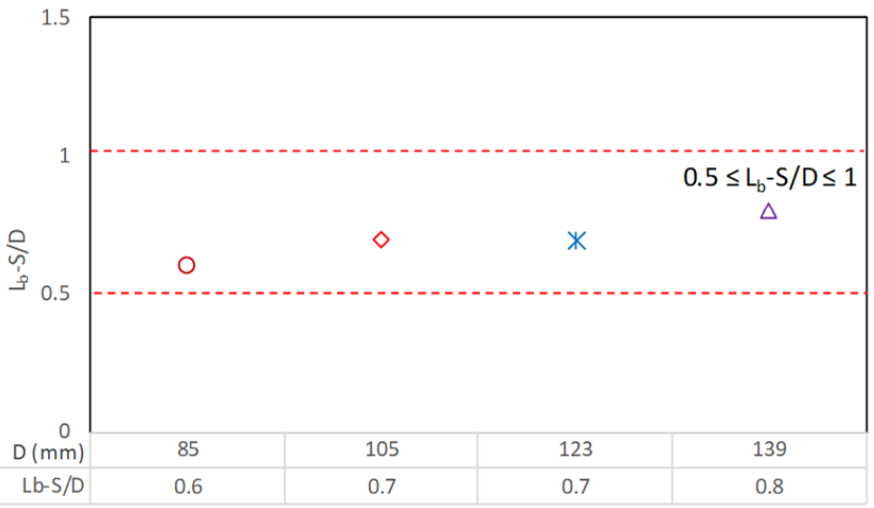

(c)

Fig. 7 The axial dimensions ratio of different cyclone diameter sizes (a) Lb/D (b) S/D (c) Lb-S/D.

\section{CONCLUSION}

In this study, the new dimensions and actual size of a unit MRdeDuster cyclone were discussed and assessed. The diameter adopted in designing the MR-deDuster unit is $105 \mathrm{~mm}$ and its other configurations is based on Dimension 2 which met all the necessary selection requirement. The predicted cut diameter and pressure drop of the selected dimension are $1.7 \mu \mathrm{m}$ and $8.6 \mathrm{~cm}$ (3.4 inches) of water respectively, which indicates that the unit is presumably able to capture fine particulates (PM2.5 and lower). Meanwhile, the axial ratio of the final design are $\mathrm{Lb} / \mathrm{D}=1.6, \mathrm{~S} / \mathrm{D}=1$ and $\mathrm{Lb}-\mathrm{S} / \mathrm{D}=0.7$, which are all within the optimum axial ratio range. The MR-deDuster was designed with the identical small body diameter and high body length miniature cyclone which results in similar performance (cut diameter, collection efficiency, and pressure drop) for any volumetric gas flow rate with the similar operation conditions. The numbers of miniature cyclones installed in MR-deDuster is the only parameter changes for different volumetric gas flow rate of the flue gas in order to maintain the performance of the unit. The prediction of high collection efficiency and low pressure drop of the unit depict the ability of the unit to serve as a good particulate emission separator in air pollution control. However, the ability of the unit will not only be highlighted in the selection of optimum configurations and prediction of the unit performance. The performance of MR-deDuster will be further verified via experimental validation.

\section{ACKNOWLEDGEMENT}

The authors would like to acknowledge the support from UTM PDRU Project with Cost Center No. R.K430000.7743.4J013 and UTM PDRU Project with Cost Center No. Q.J13000.2622.12J53 provided by Universiti Teknologi Malaysia and the Malaysian Government.

\section{REFERENCES}

[1] Norelyza, H., Rashid, M., Hajar, S., and Nurnadia, A. 2014. MRdeDuster: A dust emission separator in air pollution control. Jurnal Teknologi (Sciences \& Engineering). 68(5): 85-88.

[2] Rashid, M., Huda, N., Norelyza, H., and Hasyimah, N. 2015. Comparison of the performance of MR-deDuster with other conventional cyclones. Sains Malaysiana. 44(4): 565-569.

[3] Norelyza, H., and Rashid, M. 2013. Performance of MR-deDuster: A case study of a palm oil mill plant. Advanced Materials Research. 664: 133137.

[4] Cooper, C. D., and Alley, F. C. 2011. Air pollution control: A design approach. Waveland Press: Long Grove, IL.

[5] Theodore, L., and Buonicore, A. J. 1976. Air pollution control equipment, Vol. 1: Particulates. CRC Press: Boca Raton, Florida.

[6] Alexander, R. M. 1949. Fundamentals of cyclone design and operation. Proceeding Australasian Institute of Mining and Metallurgy. 152(3): $152-$ 153.

[7] Karagoz, I., Avci, A., Surmen, A., and Sendogan, O. 2013. Design and performance evaluation of a new cyclone separator. Journal of Aerosol Science. 59: 57-64.

[8] Hsu, C. W., Huang, S. H., Lin, C. W., Hsiao, T. C., Lin, W. Y., and Chen, C. C. 2014. An experimental study on performance improvement of the stairmand cyclone design. Aerosol and Air Quality Research. 14(3): 1003-1016.

[9] Lapple, C. E. 1951. Processes use many collector types. Chemical Engineering. 58(5): 144-151.

[10] Licht, W. 1988. Air pollution control engineering: Basic calculations for particulate collection (Vol. 10). CRC Press: Boca Raton, Florida.

[11] Theodore, L. 2008. Air pollution control equipment calculations. John Wiley \& Sons: Hoboken, New Jersey.

[12] Zhu, Y., and Lee, K. W. 1999. Experimental study on small cyclones operating at high flowrates. Journal of Aerosol Science. 30(10): 13031315.

[13] Avci, A., and Karagoz, I. 2003. Effects of flow and geometrical parameters on the collection efficiency in cyclone separators. Journal of Aerosol Science. 34(7): 937-955.

[14] Azadi, M., and Azadi, M. 2012. An analytical study of the effect of inlet velocity on the cyclone performance using mathematical models. Powder Technology. 217: 121-127.

[15] Elsayed, K., and Lacor, C. 2011. The effect of cyclone inlet dimensions on the flow pattern and performance. Applied Mathematical Modelling. 35(4): 1952-1968.

[16] Stairmand, C. J. 1951. The design and performance of cyclone separators. Transactions of the Institution of Chemical Engineers. 29: 356-383.

[17] Swift, P. 1969. Dust control in industry. Dust Control Equipment Limited: England.

[18] Norelyza, H., and Rashid M. 2013. Comparative fractional efficiency prediction of MR-deDuster. MJIIT-JUC International Symposium, 2013. Tokai University, Hiratsuka.

[19] Shepherd, C. B., and Lapple, C. E. 1940. Flow pattern and pressure drop in cyclone dust collectors cyclone without intel vane. Industrial \& Engineering Chemistry. 32(9): 1246-1248.

[20] Iozia, D. L., and Leith, D. 1990. The logistic function and cyclone fractional efficiency. Aerosol Science and Technology. 12(3): 598-606.

[21] Chen, J., and Shi, M. 2007. A universal model to calculate cyclone pressure drop. Powder Technology. 171(3): 184-191.

[22] Xiang, R. B., and Lee, K. W. 2001. Exploratory study on cyclones of modified designs. Particulate Science and Technology. 19(4): 327-338.

[23] Buttner, H. 1988. Size separation of particles from aerosol samples using impactors and cyclones. Particle \& Particle Systems Characterization. 5(2): 87-93

[24] Surmen, A., Avci, A., and Karamangil, M. I. 2011. Prediction of the maximum-efficiency cyclone length for a cyclone with a tangential entry. Powder Technology. 207(1): 1-8.

[25] Ramachandran, G., Leith, D., Dirgo, J., and Feldman, H. 1991. Cyclone optimization based on a new empirical model for pressure drop. Aerosol Science and Technology. 15(2): 135-148.

[26] Kim, G. N., Choi, W. K., and Jung, C. H. 2007. The development and performance evaluation of a cyclone train for the removal of contaminated hot particulate in a hot cell. Separation and Purification Technology. 55(3): 313-320. 